\title{
Conditions for the evolution of mimicry
}

\author{
Daniel W. Franks and Jason Noble \\ Informatics Research Institute, School of Computing, University of Leeds \\ Leeds LS2 9JT, UK. Email: \{dwfranks; jasonn\}@comp.leeds.ac.uk
}

\begin{abstract}
Poster: Mimicry is a poorly understood phenomenon; we present a simulation of the evolution of both Batesian (parasitic) and Müllerian (mutualistic) mimicry. In the model, multiple species are preyed upon by a single abstract predator; the appearance of each prey species can evolve but their palatability is fixed. Batesian mimicry evolves regardless of the initial phenotypic distance between the two species, whereas Müllerian mimicry requires an initial resemblance, except when a third (Batesian) species is present.
\end{abstract}

Mimicry is a textbook example of evolutionary adaptation. However, most existing work on mimicry is empirical and, as a result, its evolutionary dynamics are still quite poorly understood. Current models of mimicry tend to focus on the selective pressures on prey brought about by the particular learning abilities of the predator, and employ simple Monte Carlo or mathematical approaches (see e.g., Turner \& Speed, 1996; Huheey, 1988). Although predator learning is an important factor, it is also useful to look directly at the coevolution of mimetic species and their models. To that end, we present a simulation model that explores the conditions for the evolution of various types of mimicry (as far as we know, this is the first such model in the adaptive behaviour literature).

Mimicry is typically classified as either Batesian or Müllerian. Unpalatable species - many insects and snakes are good examples - often evolve conspicuous warning colouration (aposematism) which exploits predators' sensory systems such that an association is rapidly learned between the species' appearance and unpalatability. The evolution of Batesian mimicry takes advantage of aposematism in order to deceive the predator: the tasty mimic comes to resemble the unpalatable model. This is a parasitic relationship between the mimic and the model, and, because the selective pressures on the mimic are greater than those on the model, the mimic is generally expected to keep up in the resulting coevolutionary arms race Turner (1995). An example of a Batesian mimic is the hover-fly, for which the bee and the wasp are models.

Müllerian mimicry, on the other hand, is mutualistic. Two unpalatable species converge on each other's ap- pearance to gain extended protection from the predator. Thus, the predator's learning system has been exploited so that it can learn to avoid the warning colouration more quickly. The predators, of course, need to sample some of the co-mimics in order to learn to avoid them. Thus, the main advantage to an individual here is that they have less chance of being sampled by the predator, as they are protected by extra numbers. Bees and wasps are good examples of Müllerian mimicry.

\section{The Simulation Framework}

Multiple populations of prey species were used in each experiment. Different species of prey were each assigned a fixed palatibility level. Each individual had a single gene: a value representing their external appearance or phenotype. The phenotypes were constrained to a 'ring' of values from 1-20 (where 20 and 1 are neighbours). The distance of one phenotype from another represents their level of similarity.

A single, abstract predator was modelled with a simple reinforcement learning system. The predator's experience of each phenotype was represented by a score; after eating prey of a particular external phenotype, the predator would update the relevant score according to the palatibility of the individual consumed. The predator generalised on the basis of experience, and thus would also, to a lesser extent, update its scores for the four closest neighbour phenotypes. Each predator memory score had a fixed upper and lower bound, and gradually degraded back towards its starting level (usually zero, representing ambivalence).

In each generation the predator was presented with 30 binary forced-choice situations. Two individuals were randomly selected from across all prey populations present and the predator would make a probabilistic choice based on its experience of each phenotype. Random asexual reproduction then took place amongst the surviving prey. Mutation was implemented as a change of \pm 1 in the appearance or phenotype gene, and the mutation rate was 0.03. All of the experiments were run over 5000 generations, and prey species populations kept constant at 100 . The main variable manipulated was the starting distance between prey species' phenotypes, in order to determine whether an initial chance resemblance is required for the evolution of mimicry. 


\section{Results}

Figure 1 shows the results of two experiments in terms of the initial and final distances between prey species' phenotypes. Experiment 1 was conducted using one palatable and one unpalatable species. Regardless of the starting distance between the two, Batesian mimicry evolved: the palatable species came to have the same or very similar phenotype to the unpalatable one. This was also true when the predator's learning did not involve generalisation.
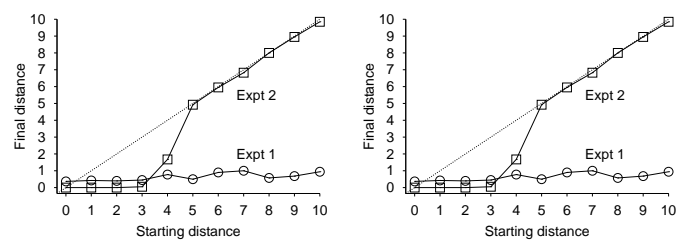

Figure 1: Final distance by initial distance between two prey species' phenotypes; each point is averaged over 40 runs. Dashed line shows zero change in phenotype.

Experiment 2 was conducted in the same way with two unpalatable species. Figure 1 shows that Müllerian mimicry only evolves if the two prey species have some initial resemblance. If they are initially more than about 4 units away on the phenotypic ring, they typically remain distinct in appearance. Further experiments were carried out in which additional, neutrally palatable species were added to the contexts of experiments 1 and 2 , in order to simulate a more diverse ecosystem. This had no qualitative effect on the results.

In experiment 3 (no graph shown) simultaneous Müllerian and Batesian mimicry was investigated by including two unpalatable and one palatable species. The results showed that the phenotype of the palatable species moved towards that of one of the unpalatable species (i.e., Batesian mimicry). Interestingly, this in turn drove the model species around the phenotypic ring and resulted in Müllerian mimicry with respect to the second unpalatable species, regardless of their initial phenotypic distances.

\section{Conclusions and Further Work}

Experiment 1 shows that Batesian mimics will close in on the model regardless of how different their initial phenotypes are, and regardless of the predator's ability to generalise. This shows that there is more selective pressure for the palatable species to resemble the model than there is for the model to diverge. However, there is an additional reason why the mimic is successful in "catching" the model: before mimicry has evolved, palatable individuals gain an inherent fitness benefit for mutating away from the modal phenotype of their species. This is because, when presented with a choice, the predator would be more likely to select a well-known phenotype than a newer one.

The results of experiment 2 show that Müllerian mimicry relies on an initial resemblance between the species involved. Such initial resemblance might be caused by any number of factors, such as sexual selection, random drift, etc. (It should be noted that the particular resemblance threshold found in the experiment - four units on the phenotypic ring - is of course determined by the nature of the predator's generalisation.) Experiment 2 has a somewhat paradoxical outcome in that the co-mimics, once resemblance is achieved, do not gain any immediate fitness benefit from their resemblance, at least in the absence of alternative prey species. This is because the predator must always eat one of the two choices presented to it. However, the paradox is resolved when we note that a mutant individual would represent a novel and thus appealing meal for the predator.

Experiment 3 demonstrates that pressure due to Batesian mimicry can be a force that drives Müllerian mimics together despite a lack of initial resemblance. This may constitute an explanation for the poorly understood phenomenon of "mimicry rings", in which many Müllerian mimics share a common appearance - the origin of such rings from divergent beginnings is a mystery to biologists.

Clearly, one weakness of the current model is that phenotypes are expressed in a single dimension. Future work will incorporate a richer, multi-dimensional phenotypic space, as well as a non-trivial mapping between genotype and phenotype (the latter is an issue often overlooked in evolutionary simulation modelling). More realistic predation could be introduced by modelling predator perception and learning using neural networks, and allowing the predator to coevolve with the prey, as in nature. Previous work in biology suggests that prey frequency, prey density, predator diversity and the evolution of aposematism itself are factors also worth including in a more complete model.

\section{References}

Huheey, J. (1988). Mathematical models of mimicry. In American Naturalist, p. 131.

Turner, J. (1995). Mimicry as a model for coevolution. In Arai, R., Kato, M., \& Doi, Y. (Eds.), Biodiversity and Evolution, pp. 131-150. Tokyo: National Science Museum Foundation.

Turner, J., \& Speed, M. (1996). Learning and memory in mimicry. i. simulations of laboratory experiments. In Phil. Trans. Royal Society London. Tokyo: National Science Museum Foundation. 